\title{
Prendre la parole en révolution le cas Palloy, démolisseur de la bastille
}

Speaking up during the Revolution. The case of Palloy, the demolition contractor of the Bastille

\section{Héloïse Bocher}

\section{(2) OpenEdition}

\section{Journals}

\section{Édition électronique}

URL : https://journals.openedition.org/ahrf/13164

DOI : 10.4000/ahrf.13164

ISSN : 1952-403X

Éditeur :

Armand Colin, Société des études robespierristes

Édition imprimée

Date de publication : 1 juin 2014

Pagination : 81-106

ISBN : 978-2-908327-68-7

ISSN : 0003-4436

\section{Référence électronique}

Héloïse Bocher, «Prendre la parole en révolution le cas Palloy, démolisseur de la bastille », Annales

historiques de la Révolution française [En ligne], 376 | avril-juin 2014, mis en ligne le 01 juin 2017, consulté le 01 juillet 2021. URL : http://journals.openedition.org/ahrf/13164 ; DOI : https://doi.org/ 10.4000/ahrf. 13164 


\title{
PRENDRE LA PAROLE EN RÉVOLUTION \\ LE CAS PALLOY, DÉMOLISSEUR \\ DE LA BASTILLE
}

Héloïse BOCHER

\begin{abstract}
Comprendre les origines du mythe de la Bastille et les conditions de la construction de cette mémoire nécessite de revenir sur le moment crucial que constitue l'« après » de la prise, en particulier sur le rôle du démolisseur de la prison, Pierre-François Palloy et sur les ressorts de son action. Les conditions de sa prise de parole, par le biais de fêtes, cérémonies, discours, mais aussi par la multiplication de dons d'objets divers, constitueraient une nouvelle façon d'exercer un pouvoir sur la scène publique et d'imposer un discours et une mémoire.
\end{abstract}

Mots-clés : Révolution, Bastille, mémoire, mythe, réseau, pouvoir, langage, objet, sans-culotte, Paris.

Aujourd' hui notre fête nationale connaît une organisation clairement ritualisée. Tout d'abord les Français assistent à un défilé militaire sur les Champs-Élysées, moment officiel de la démonstration de force d'une République unie derrière ses armées ; puis vient le moment d'une fête plus populaire, familiale et spontanée, faite de feux d'artifices, de bals et de retraites aux lampions. Derrière cette organisation se cache pourtant une célébration qui, par son origine, reste bien ambiguë. Si le 14 juillet fait bien référence à la prise de la Bastille, reste à savoir cependant si nous fêtons la prise elle-même du colosse ou bien sa commémoration apaisée, à travers la fête de la Fédération de 1790. En revenant à la journée même de la prise de la forteresse et en la comparant à son souvenir, au symbole qui y a été associé, force est de constater le hiatus qui existe entre le fait (la prise de la Bastille par les Parisiens) et l'événement aujourd'hui célébré. 
Au soir même de la prise de la Bastille plusieurs centaines d'ouvriers, munis de pioches, sont lancés dans une sorte de deuxième assaut contre le colosse. Une première mémoire et, par là même, une première histoire de la Bastille et de la Révolution s'écrivent alors « en direct », dont il s'agit ici d'analyser les acteurs et les enjeux. À cette occasion entre en scène Pierre-François Palloy, démolisseur de la forteresse, dont l'action va participer pleinement à l'écriture du mythe. Il s'agira donc d'étudier le parcours de cet homme ainsi que les ressorts de son action, en observant les modalités de sa prise de parole publique, afin d'identifier les nouvelles voies d'affirmation d'une influence sociale, sinon d'un pouvoir politique.

Pour comprendre les origines du mythe de la Bastille, il convient de rappeler tout d'abord la force symbolique de la forteresse. La Bastille, par son allure imposante à l'entrée même du quartier populaire et ouvrier du faubourg Saint-Antoine, fait figure de monument à la fois craint et honni par le peuple de la capitale. En outre, par son statut de prison d'État la forteresse sert de principal lieu de détention de tous ceux qui sont sensés mettre en danger la sûreté de l'État. Enfermés par simples « lettres de cachet », ceuxci apparaissent comme les victimes d'un système qui symbolise l'arbitraire monarchique. En effet, les lettres de cachet, instruments privilégiés de la volonté personnelle du souverain, font office de décision de justice. Assorties d'une obligation de secret sur l'identité du prisonnier, sur le motif de son arrestation et sur les conditions de sa détention, elles font de la Bastille l'objet de récriminations et de dénonciations de plus en plus féroces tout au long du XVIII ${ }^{\mathrm{e}}$ siècle. Or, cette image de la prison joue un rôle majeur dans la diffusion de l'idée d'une nécessaire démolition de la forteresse. Par là, il s'agit à la fois de détruire une prison dont l'utilité, par les frais qu'elle engage, est remise en cause, mais surtout d'anéantir le symbole d'un système politique désormais affaibli.

Ainsi, l'idée de démolir la Bastille ne naît pas au soir du 14 juillet. À mesure que la réflexion se renouvelle dans les domaines de la philosophie politique et du droit de multiples projets de démolition voient le jour tout au long du XVIII ${ }^{\mathrm{e}}$ siècle. Indice de cette évolution des réflexions politiques, les ingénieurs et architectes de l'époque proposent divers plans de réaménagement de la place dans l'hypothèse de la disparition de la forteresse ${ }^{1}$. Reste à passer à l'acte. Celui-ci sera engagé dans les quelques

(1) L'architecte Corbet, inspecteur des bâtiments de la Ville propose, dès 1784, l'aménagement d'une place où serait érigée en son centre une statue de Louis XVI ceinturée de nouveaux bâtiments d'où partiraient plusieurs rues et boulevards. Ce projet de monument en l'honneur du roi se retrouve également chez le magistrat Davy de Chavigné qui, en juin 1789, soumet à l'Académie royale 
heures qui suivent la prise de la prise de la Bastille par les Parisiens le 14 juillet. Se joue alors le deuxième temps de l'écriture du mythe. Il convient en effet de revenir sur le moment crucial que constitue cet « après » de la prise. Des analyses riches et originales ont déjà été produites sur ce processus d'écriture du symbole et de la mémoire de l'événement ${ }^{2}$. Il ne s'agit en aucun cas de revenir sur ces études qui permettent d'éclairer le sens et les origines de cet «événement symbole », ou de rappeler les conditions de l'écriture du mythe de la Bastille. Ces auteurs ont en effet dévoilé les principaux éléments explicatifs de la construction du mythe que l'on connaît aujourd'hui. Plus précisément, ils ont insisté sur l'importance, dans ce processus, de la matérialité du souvenir. Matérialité qui les amène à souligner alors le rôle de Palloy, protagoniste inévitable de la construction d'une nouvelle sacralité, de la définition d'une nouvelle théâtralité des pratiques commémoratives et de nouvelles stratégies de prise de parole dont l'aboutissement est une prise de pouvoir originale qui mérite toute notre attention. C'est précisément sur le rôle de Palloy et sur les ressorts précis de son action que nous souhaiterions revenir dans cet article, afin de montrer que ce rôle ne peut être résumé à une «activité fiévreuse » ne répondant qu'à une tendance monomaniaque teintée d'opportunisme et de mythomanie (ce à quoi Palloy a été trop souvent réduit...). Au contraire, peut-être serait-il plus judicieux de considérer cette prise de parole comme une nouvelle façon de s'affirmer sur la scène publique, une nouvelle façon d'imposer un discours et une mémoire.

Pour comprendre ce processus d'écriture de la Révolution par ellemême, et ceci dès les premiers instants, il nous faudra donc revenir sur le rôle joué par Pierre-François Palloy, maître-maçon et démolisseur de la Bastille. Celui-ci, en effet, nous permet à la fois de saisir comment la journée du 14 juillet s'est hissée au rang de symbole incontournable de la mythologie révolutionnaire mais aussi, par ses modalités d'action, de saisir comment les nouveaux supports d'un discours public, les nouvelles façons d'affirmer une position sociale lui ont permis d'exercer un pouvoir

d'architecture un projet de monument à ériger en l'honneur de Louis XVI « libérateur » sur les ruines de la Bastille.

(2) Hans-Jürgen LÜSEBRINK, Rolf REICHARDT, The Bastille: A History of a Symbol of Despotism and Freedom, Duke University Press, 1997. Voir aussi Hans-Jürgen LÜSEBRINK et Rolf REICHARDT, « Die "Bastille". Zur Symbolgeschichte von Herrschaft und Freiheit », Annales Histoire, Sciences Sociales, 1995, vol. 50, n 4, p. 814-816. Sur la question de la définition de l'« événement » voir Paul RicoEUR, «Événement et sens », Raisons pratiques, 1991, n², ou encore Pierre NORA, «Le retour de l'événement », Faire de l'histoire, 1974, Paris, Gallimard, t.1. Sur l'imagerie de la Bastille : Rolf REICHARDT, L'imagerie révolutionnaire de la Bastille, Collections du Musée Carnavalet, Paris, 2009. 
et de faire de la politique. En ce sens, à travers la création du symbole de la Bastille qui nous est parvenu, Palloy serait paradigmatique d'une nouvelle vie politique et publique qui se met en mouvement dès les premiers temps de la Révolution. Afin de mettre à jour cette première écriture de l'histoire et de la mémoire de la Révolution, nous reviendrons donc sur le rôle joué par Pierre-François Palloy. Véritable créateur du symbole de la Bastille, le démolisseur parvient, à travers de nombreux supports (multiples fêtes, maquettes, discours...) $)^{3}$, à opérer une prise de parole tout à fait efficace qui mérite que l'on en étudie les ressorts et les modalités.

\section{Quelles sources ?}

L'historiographie a bien sûr largement décrit le déroulement de la journée du 14 juillet mais l'épisode très particulier qu'est la démolition de la forteresse a été largement passé sous silence, comme si une parenthèse s'ouvrait au soir du 14 juillet 1789 , pour ne se rouvrir qu' au XIX ${ }^{\mathrm{e}}$ siècle avec la toute nouvelle fête nationale instituée par la $\mathrm{III}^{\mathrm{e}}$ République. La Bastille de 1789 ayant en quelque sorte disparu pour ne laisser place qu'au symbole digne de la fête nationale ${ }^{4}$. Pourtant, un corpus particulièrement riche et varié nous permet de mettre à jour ce moment précis, crucial, des premiers temps de l'écriture de la mémoire de la prise et de la démolition de la Bastille. Il ne s'agit pas ici de dresser le panorama complet des sources, mais de présenter les fonds qui permettent d'approcher précisément la figure de Palloy, son activité autour du chantier et les procédés qu'il utilise pour « écrire » la Bastille ${ }^{5}$. En premier lieu, la Bibliothèque historique de la Ville de Paris (BHVP) conserve à la cote CP 5252 une grande partie de la correspondance de Palloy. Nous y trouvons des lettres concernant la démolition de la Bastille et les divers dons d'objets souvenirs et de maquettes de la forteresse que le démolisseur a pu faire. Nous y rencontrons donc la correspondance que Palloy adresse à l'Assemblée nationale, à la

(3) Sur les fêtes organisées par Palloy et l'architecture de l' « éphémère »: Werner SzAMBIEN, Les projets de l'an II. Concours d'architecture de la période révolutionnaire, Paris, 1986.

(4) Fernand BouRnon, La Bastille (Histoire générale de Paris), Imprimerie nationale, 1893 ; Guy CHAUSSINAND-NOGARET, 1789, la Bastille est prise : la Révolution française commence, Bruxelles, Éditions Complexe, 1988 ; Monique COTTRET, La Bastille à prendre, Histoire et mythe de la forteresse royale, PUF, 1986 ; Jacques GODECHOT , La prise de la Bastille : 14 juillet 1789, Paris, Gallimard, 1965 ; Claude QuETEL, L'Histoire véritable de la Bastille, Paris, Larousse, " Bibliothèque historique », Larousse, 2006.

(5) Pour une histoire des archives de la Bastille : Émilie DuTRAY-LECOIN, Danièle MuZERELLE (dir.), La Bastille ou « l'enfer des vivants » à travers les archives de la Bastille, catalogue de l'exposition présentée à la Bibliothèque nationale de France à la bibliothèque de l'Arsenal, du 9 novembre 2010 au 11 février 2011, BnF, 2010. 
Municipalité parisienne, aux sections, districts et départements, mais aussi aux particuliers afin de présenter son entreprise de démolition et d'offrandes. Nous trouvons également dans ce fonds de nombreuses lettres traitant des cérémonies, processions et fêtes que Palloy a pu organiser à partir ou hors de son chantier. La BHVP conserve également des cahiers où est rassemblée la correspondance de Palloy recopiée par ses secrétaires. De la cote CP 5253 à 5260 sont conservés les « registres » de Palloy, couvrant les années 1790 à 1792 . Il s'agit de cahiers manuscrits de 350 feuillets chacun environ. Ce corpus présente cependant d'importantes lacunes puisque sur un total de 23 cahiers, seulement 9 ont été conservés. Cette correspondance recopiée a posteriori, avec toutes les limites que ce genre de document peut présenter, se révèle néanmoins particulièrement prolixe. Notons à ce titre que le département des manuscrits de la Bibliothèque nationale conserve le premier de ces 23 cahiers, qui apparaissait comme manquant à la BHVP. L'existence de ce cahier est particulièrement importante puisque celui-ci rend compte de l'activité de Palloy dès le 14 juillet 1789, et donne ainsi de précieuses informations sur les modalités de la mise en place immédiate du chantier de démolition de la Bastille, comme sur les relations que Palloy entretient avec les multiples acteurs de ces journées éminemment décisives pour la suite de son itinéraire. Ces cahiers présentent tous à peu près la même forme et les mêmes types de documents. Ils reproduisent, tout d'abord, la correspondance que Palloy a jugée utile de présenter à l'Assemblée. Ceci explique en partie que nous n'y trouvions quasiment pas de courrier à caractère privé. Les lettres font donc essentiellement référence au rôle public, politique et professionnel de Palloy, à ses affaires d'ordre financier et contractuel. S'y trouvent également de nombreuses lettres témoignant d'intenses échanges entre Palloy et les autorités publiques. Nombreuses aussi sont les lettres qui concernent encore une fois les cérémonies qu'il a pu organiser et les envois qu'il a pu faire. Ces cahiers enregistrent également une abondante production de papiers de comptes, notamment la totalité des « rôles de payes » des équipes affectées à la démolition de la Bastille. Ce corpus complet, de la $1^{\text {re }}$ paie (le 15 juillet 1789) à la $94^{\mathrm{e}}$ (le 7 mai 1791), fourmille ainsi d'informations multiples sur l'organisation quotidienne du chantier. De même sont disponibles les comptes tenus par Palloy à l'occasion de l'organisation de fêtes et de cérémonies. Ces papiers donnent à voir les divers postes de dépenses et le montant des sommes mobilisées. ${ }^{6}$ 
Pour compléter ce corpus manuscrit de Palloy il convient de mentionner également un nombre important de documents conservés à la Bibliothèque municipale de Sceaux ${ }^{7}$, au Musée de l'Île-de-France ${ }^{8}$ et aux Archives nationales ${ }^{9}$. Ces lettres de Palloy portent sur divers thèmes, mais la correspondance liée à la démolition de la Bastille et à ses multiples dons est là encore largement surreprésentée. L'action publique de Palloy sur la scène parisienne peut, quant à elle, être abordée par l'intermédiaire du fonds des archives de Paris et des Archives nationales concernant les sections. Nous renvoyons ici en particulier aux travaux d'Albert Soboul ${ }^{10}$. Aux Archives nationales, la série $\mathrm{F} 7$, qui totalise les registres des procès-verbaux des assemblées générales et des comités révolutionnaires de 22 sections, peut constituer une première piste pour tenter d'analyser la place que Palloy a pu tenir au sein de sa section. En outre, les archives de Paris conservent, dans la série VD*, les papiers de la Commune de Paris ${ }^{11}$. Les travaux de Maurice Tourneux ${ }^{12}$ et de Sigismond Lacroix ${ }^{13}$ sont par ailleurs deux instruments de travail particulièrement utiles pour appréhender ce fonds. À ce riche fonds de manuscrits il convient d'ajouter un très important fonds imprimé conservé à la Bibliothèque nationale François Mitterrand, Palloy ayant publié un total de 154 imprimés sous la forme de discours et de divers fascicules.

Il convient également de présenter les sources qui permettent d'approcher un autre aspect du rôle de Palloy, celui de concepteur et de producteur de médailles. Celles-ci mettent en scène et commémorent la démolition de la forteresse et figurent ainsi parmi les supports privilégiés de la mise en valeur par Palloy de son chantier et de son action. Faisant l'objet de fortes convoitises parmi les numismates, ce fonds est extrêmement dispersé. Les collections privées, difficiles d'accès, constituent donc une source essentielle pour tenter d'appréhender ce corpus dans sa totalité. Dans ce contexte,

(7) Bibliothèque municipale de Sceaux, mss 005 à ms 070.

(8) Musée de l'Île-de-France, Château de Sceaux, voir la cote 70.8.1 (E.3978), et les cotes allant de 89.85.1 à 89.85.16.

(9) AN, F13 1242. Démolition de la Bastille 1789-an V. Voir aussi F7/10811, Dossier Palloy, prairial an VI. Palloy demande au ministre une nouvelle carte de sûreté, lui établissant domicile à Paris.

(10) Albert SoBOul, Les papiers des sections de Paris (1790- An IV), répertoire sommaire, Paris, Maurice Lavergne imprimeur, 1950.

(11) Archives de Paris, Série VD* 1 à 89 , Fonds de la Ville de Paris, 1789-An VII.

(12) Maurice TourneuX, Procès-verbaux de la Commune de Paris (10 août 1792- $1^{\text {er }}$ juin 1793, Paris, Société de l'Histoire de la Révolution française, 1894.

(13) Sigismond LACROIX, Actes de la Commune de Paris pendant la Révolution, Index des noms de personnes, de lieux et de matières par André Vaquier, Paris, Service des travaux historiques de la Ville, 1955, tome II. 
les ouvrages de Michel Hennin ${ }^{14}$, Alain Weil ${ }^{15}$ et Gilles Michaud ${ }^{16}$, qui proposent une représentation des coins frappés au nom de Palloy, sont particulièrement précieux pour l'historien. Néanmoins, au-delà des collections privées, certains établissements publics tels que le Musée Carnavalet, le musée de la Révolution française à Vizille, et le musée de l' ̂̂le-de-France conservent des exemplaires de médailles. Nous dénombrons aujourd'hui, selon les estimations d'Alain Weil, 45 coins dont 15 qui n'avaient pas été décrits par Michel Hennin ${ }^{17}$.

Enfin, les objets-souvenirs dont Palloy a été le concepteur peuvent nous apparaître au travers des collections des musées. Le musée Carnavalet par exemple conserve la maquette d'une Bastille sculptée ${ }^{18}$, ainsi que de nombreux objets fabriqués à l'aide de vestiges de la forteresse : un jeu de dominos et sa boîte offerts au jeune Dauphin ${ }^{19}$, une table, un couteau. Il convient également de prendre en compte les multiples pierres de la Bastille, gravées d'un plan de la forteresse et parfois de la déclaration des droits de l'Homme et de la Constitution de 1791, envoyées par Palloy dans les départements et les districts. Nous n'avons pu inventorier l'ensemble de ces pierres, nous signalons cependant celle du château de Vizille ${ }^{20}$, de même que l'eau-forte coloriée qui servait d'illustration à la pierre ${ }^{21}$ possédée par le Musée Carnavalet. Ce détour prolongé parmi les sources nous permet de prendre la mesure de l'ampleur des documents à notre disposition pour appréhender l'entreprise de Palloy et ainsi d'embrasser l'extrême richesse des documents associés au chantier de démolition de la Bastille et à la création, sur ses ruines, de sa symbolique.

Simple «fait» de l'histoire, la prise de la Bastille se transforme, en quelques heures seulement, sous les coups de pioches des ouvriers de Palloy, en véritable « événement » marquant l'entrée de la France dans une nouvelle ère politique. Revenons donc sur cette entrée en scène de Palloy au soir du 14 juillet, moment crucial de cet été 1789.

(14) Michel Hennin, Histoire numismatique de la Révolution Française, Paris, 1826. Consulter les planches du $2^{\mathrm{e}}$ volume, qui représentent les coins frappés par Palloy.

(15) Alain WeIL, Histoire et numismatique du patriote Palloy, SPES, Lyon, Antika 1, 1976. Reproductions photographiques des coins, I-XIII.

(16) Gilles Michaud, Numismatique et documents du Patriote Palloy, Feuille à Feuille Éditions, 2012.

(17) Alain WeIL, op.cit., p. 61.

(18) Musée Carnavalet, cote S. 503.

(19) Musée Carnavalet, Inv. OM. 520.

(20) Château de Vizille, MRF D.1983-1

(21) Musée Carnavalet, TOPO GC XXI A 


\section{L'entrée en scène de Palloy}

Rappelons tout d'abord que Palloy, par l'importance de son atelier, figure parmi les entrepreneurs les plus prospères de la capitale ${ }^{22}$. En 1775, à l'âge de 20 ans, Palloy entre comme commis chez François Nobillot, entrepreneur de maçonnerie rue du Mûrier dont il épouse la fille le $1^{\mathrm{er}}$ février 1776. De cette union naît Charlotte-Louise, le 12 novembre 1776 dont l'acte de naissance nous indique que la famille de Palloy s'inscrit dans la haute bourgeoisie parisienne ${ }^{23}$. En effet le parrain, Barthélémy Hébert, est qualifié de bourgeois de Saint-Germain-en-Laye, et la marraine, CharlotteGabrielle-Clothilde Desfevres, est la veuve de Pierre-Léon, docteur agrégé en droit de la faculté de Paris. François Nobillot meurt probablement vers 1781. Quelques années plus tard, Palloy loue sa maison et s'établit au n ${ }^{\circ} 20$ de la rue des Fossés-Saint-Bernard, dans un immeuble pourvu d'un vaste atelier, acheté le 20 novembre $1786^{24}$. À cette occasion, il se fait appeler dans l'acte de vente, signe de sa réussite professionnelle, « entrepreneur de bâtiments » et non plus « maître maçon » comme lors de la naissance de sa fille.

Enfin, dans un récit autobiographique manuscrit ${ }^{25}$, Palloy affirme, en 1794, que sa fortune d'avant la Révolution s'évaluait à près de 500000 livres. Ce chiffre, considérable, ne semble pas exagéré car il est alors propriétaire de deux autres maisons, rue Geoffroy-Lanier et rue Mouffetard (à l'enseigne du Paradis Terrestre, achetée 10509 livres le 4 août 1781$)^{26}$, d'une échoppe rue Saint-Victor, (payée la même année 640 livres) ${ }^{27}$, et de deux magasins, rue Traversière et rue du Bon-Puits, (ce dernier acheté 1600 livres en 1779 $)^{28}$. Il semble enfin qu'il soit propriétaire de deux terrains rue Thiroux, et place du Palais-Bourbon. Ainsi, âgé d'à peine trente-cinq ans, Palloy se trouve à la tête d'une fortune immobilière considérable. Son métier d'entrepreneur en maçonnerie lui procure en outre

(22) B.H.V.P., Cote 601 254.Il peut être qualifié d'architecte, architecte entrepreneur, artiste, mais aussi, entrepreneur, entrepreneur des Bâtiments du Roi, ou encore Maître Maçon, Maître Maçon de la Verrerie du Roy.

(23) Archives de Paris, acte de naissance reconstitué, 5 Mi 152.

(24) Archives de Paris, acte de vente de l'atelier : DC/6/130, folio 109. Pour une description de la maison rue des Fossés-Saint-Bernard : DQ16/1022, n ${ }^{\circ} 6252$.

(25) Archives de Paris, 4 AZ 719, collection Boret, Papiers Palloy.

(26) Archives de Paris, acte de vente du Paradis terrestre : DC/6/123, folio 83.

(27) Archives de Paris, acte de vente de l'échoppe rue Saint-Victor : DC/6/138, folio 125.

(28) Archives de Paris, acte de vente du magasin du Bon-Puits : DC/6/138, folio 148. 
une forte assise sociale qui amène les autorités à se tourner vers lui lors de la commande de nombreux chantiers ${ }^{29}$.

C'est cette puissance que Palloy va utiliser de façon pour le moins spectaculaire au soir même du 14 juillet en envoyant, sans autorisation aucune de la part de la Municipalité, des centaines de ses ouvriers sur la forteresse encore fumante afin de la démolir.

Alors que la prise de la Bastille apparait comme la victoire du peuple de Paris tout entier, un individu seul s'approprie le lieu et s'arroge le droit d'en disposer. Tout en mettant les Électeurs de la ville de Paris devant le fait accompli, Palloy reste néanmoins prudent et demande dans une lettre datée du 16 juillet au matin que sa fonction d'entrepreneur responsable de la démolition lui soit officiellement confirmée :

« Je vous prie, Messieurs, de vouloir bien joindre à votre proclamation un ordre de continuer la démolition, si vous me croyez capable d'exercer mon courage. Je puis vous assurer de mon exactitude, de ma fermeté et de mon dévouement que je mettrai à cette besogne, la regardant à ce moment comme le chef-d'œuvre de notre conquête ; et soyez assurés que je serai toujours pour la vie ferme et inébranlable, et pour la vie, je m'engage à ne signer que Palloy, Patriote $»^{30}$.

Le Comité permanent de l'Hôtel de Ville répond à sa demande par un arrêt validé par la Commune :

«Le comité a arrêté que la Bastille serait démolie par tous les districts ensemble, sous l'inspection cependant du district de Saint Louis la Culture,

(29) Le 21 novembre 1789, Palloy reçoit l'ordre de faire établir sur le Pont-Neuf, devant la statue d'Henri IV une plate-forme destinée à recevoir des canons, pour une somme de 1450 livres. En janvier 1790, les ouvriers de Palloy nettoient la Seine et l'égout des fossés de la Bastille. De février à août de la même année, ils bâtissent, avec des pierres de la forteresse, des postes pour la Garde Nationale au boulevard du Temple, à Saint-Louis-la-Culture, à Sainte-Opportune, et sur le boulevard de l'Opéra. Au mois de juin, Palloy se charge de la démolition de la Halle aux farines, des baraques sur le quai d'Orsay, et d'une maison au coin de la rue Cotte et du faubourg Saint-Antoine. Lors de la prise du château des Tuileries, le 10 août 1792, l'incendie se développe entre le Carrousel et le Palais. Le 12 août, sur la proposition de deux députés, Goupilleau et Chabot, l'Assemblée charge « le citoyen Palloy, homme de confiance, très propre par son talent et son civisme à rendre ce service » d'abattre les statues de Louis XIII, place Royale, d'Henri IV sur le Pont-Neuf, et de Louis XIV, place de la Révolution. Santerre lui accorde en outre un service d'ordre pour tenir la foule en respect. Palloy est également chargé des travaux mis en œuvre dans la prison du Temple pour l'arrivée du roi, le 13 août 1793.Palloy rend ses comptes le 11 septembre avant de partir aux armées.

(30) BNF Richelieu, NAF 2811, fol 21-22, copie de la lettre adressée au corps électoral séant à la ville par M. Palloy, du 16 juillet 1789 du matin. 
et que M Palloy serait prié de continuer la démolition par lui commencée, et que l'ordre lui en serait donné $»^{31}$.

Palloy est donc officiellement confirmé dans sa tâche, même si la nouvelle municipalité lui indique que la gestion des travaux sera sous la direction des architectes de la Poise, Jallier de Savault, de Montizon et Poyet.

Cette entrée en scène de Palloy mérite d'autant plus réflexion si l'on s'arrête quelques instants sur l'ampleur des travaux engagés. En effet, ce sont quasiment mille hommes qui quotidiennement vont prendre part pendant presque deux années au chantier. Nous ne pouvons ici revenir sur le détail tout à fait impressionnant de l'activité foisonnante, de la concentration d'hommes, de matériaux, et d'outils dont les sources témoignent ${ }^{32}$. En effet, les fonds constitués des papiers de comptes et de la correspondance de Palloy mettent en scène maçons, compagnons, commis, chefs, souschefs et inspecteurs, nous offrant ainsi un aperçu de l'organisation des hommes évoluant sur le site. Dans son discours de clôture de sa mission, Palloy revient très précisément sur le fonctionnement quotidien du chantier, tâchant par là de prouver son efficacité et sa rigueur :

« Après la prise de la Bastille, il fut établi un ordre qui a été régulièrement observé, les ouvriers qui ont été employés à la démolition de cette forteresse, furent placés par classe d'ateliers en nombre égal d'hommes sous l'inspection de leurs sous-chefs, lesquels ateliers étaient inspectés par des chefs qui avaient une certaine quantité de sous-chefs sous leur surveillance. Les rôles se faisaient strictement, et tous les jours l'appel nominal était fait par mon commis qui vérifiait les feuilles de chaque atelier et communiquait la feuille générale aux inspecteurs nommés par les architectes qui certifiaient par des nouveaux appels ces feuilles de rôles, qui ensuite étaient remises au Bureau de la Bastille tous les soirs, ces mêmes feuilles étaient visées par les Inspecteurs et de là portées au bureau du comité permanent des Électeurs depuis le 16 juillet $1789 »^{33}$.

Ainsi, dès la nuit du 14 au 15 juillet 1789, un nouveau paysage et un nouveau microcosme apparaissent, faisant du quartier de la Bastille un nouveau centre parisien, un lieu où se joue désormais « en direct » ce qui

(31) BNF Richelieu, NAF 2811, f ${ }^{\circ}$ 23, arrêté du Comité permanent, 16 juillet 1789.

(32) Pour cette étude précise, voir Héloïse BOCHER, Démolir la Bastille. L'édification d'un lieu de mémoire, Paris, Éditions Vendémiaire, 2012.

(33) Extrait du Discours préliminaire de Palloy, BN, NAF 2811. 
va devenir un des épisodes essentiels de l'histoire de la Révolution. Le chantier constitue à la fois un tremplin pour Palloy, qui, par le chantier, devient une figure incontournable de la vie publique, mais surtout l'endroit qui va servir de base à l'écriture de la Révolution, la Bastille devenant peu à peu, « en direct », le symbole que l'on connaît aujourd'hui. Reste à tenter de comprendre en quoi Palloy, par son omniprésence sur la scène publique, participe très activement à l'écriture d'une première mémoire de la Bastille.

\section{Une figure omniprésente}

Il convient tout d'abord de souligner le vif succès que connaît le chantier de la Bastille. Les visites organisées s'y succèdent où l'on reconnait des figures aussi célèbres que celles de Mirabeau, du marquis de Lusignan ou encore de Beaumarchais. Ces visites témoignent du vif attrait des Parisiens pour ce chantier, véritable matérialisation de la fin d'un monde. En parcourant les moellons de la forteresse abattue, en se faisant enfermer pour la nuit dans les cachots, les visiteurs ont le sentiment de participer à l'écriture de l'histoire tout en témoignant de leur désir de vivre, de ressentir cette transition. On observe donc dans ces nouveaux usages du quartier ce qu'Éric Fournier a appelé la poétique des ruines, la fascination opérée sur ceux qui souhaitent ressentir le frisson que pouvaient connaître les prisonniers d'un monde que l'on veut croire révolu ${ }^{34}$. Palloy participe activement à cette orchestration de la célébrité de ce qui apparaît comme « son » œuvre et organise lui-même des processions afin d'alimenter ce succès. Par ses fêtes, ses cérémonies, ses multiples discours publics, Palloy apparaît comme une figure incontournable et omniprésente du thêâtre politique et culturel parisien, comme en témoignent par exemple les bals qu'il organise sur les ruines de la Bastille, devenus lieu d'une nouvelle sociabilité urbaine patriotique ${ }^{35}$.

Ainsi, le 14 août 1789, un mois après les premiers coups de pioche, 500 ouvriers se rendent au Palais-Royal pour y promener, comme des trophées, trente-sept boulets trouvés dans un des murs de la Bastille. Quelques mois plus tard, le 22 février 1790, Palloy organise une grande pompe, qui s'achève le lendemain par une procession des ouvriers du

(34) Éric Fournier, Paris en ruines. Du Paris haussmannien au Paris communard, Imago, 2008.

(35) Voir la Vue de la décoration et illumination faite sur le terrain de la Bastille pour le jour de la fête de la Confédération française le 14 juillet 1790, Aquatinte coloriée, Musée Carnavalet, G64123. 
chantier jusqu'à l'Hôtel de Ville, à l'occasion de laquelle un modèle de la Bastille sculpté dans une pierre de la forteresse porté en triomphe est solennellement déposé dans la maison commune. Entre autres célébrations, retenons enfin l'épisode de la découverte dans les ruines de la prison de squelettes que l'on veut considérer comme les ossements de malheureuses victimes de l'Ancien Régime. Cet épisode souligne à quel point Palloy dépasse son statut technique de démolisseur pour se draper des attributs du libérateur. Il invite au mois de mai 1790 le tout Paris à se recueillir devant les dépouilles avant d'organiser le $1^{\text {er }}$ juin leur inhumation en grande pompe au cimetière Saint Paul ${ }^{36}$. À cette occasion Palloy rédige l'épitaphe du monument, fait de pierres de la Bastille, dressé à cette occasion :

« Sous les pierres mêmes des cachots

Où elles gémissaient vivantes

Reposent en paix

Quatre victimes du despotisme !

Leurs os découverts

Et recueillis par leurs frères libres

Ne se lèveront plus

Qu'au jour des justices

Pour confondre leurs tyrans $»^{37}$

Cette omniprésence de Palloy, sa capacité à investir la capitale par l'intermédiaire de son entreprise de démolition est de plus clairement illustrée par le foisonnement étonnant de sa prise de parole publique, notamment devant ce lieu essentiel qu'est l'Assemblée nationale. Discours, poèmes, tous les événements de la vie politique parisienne et nationale sont l'occasion pour Palloy de se mettre en scène et de rappeler sa tâche de démolisseur. À titre d'exemple, à l'automne 1793, alors que les comptes du chantier ont été rendus depuis le 21 mars 1792, Palloy continue d'utiliser son œuvre sur le chantier pour exprimer maintenant son attachement à la République. Auteur d'un Serment républicain qui accompagne ses dons de médailles, Palloy écrit dans le registre épique qui lui est caractéristique :

(36) Les 4 et 7 mai, et 12 juin 1790. Les cadavres dont les os sont renfermés sous celle Tombes ont été découverts dans la démolition des souterrains des Cachots de la Bastille et transportés au cimetière de l'Eglise St Paul dans une Bierre mise sous ce tombeau d'après les soins de Pierre François Palloy, Patriote qui l'a fait graver à ses frais. Aquatinte par Lebas, 1790, 18,9 x 25, 6 cm, BnF, Estampes, Histoire de France, cliché M 99918.

(37) Archives de Paris, 4 AZ 719, collection Boret, Papiers de Palloy. 
« Nous promettons, en Républicains, que nous exterminerons tous les tyrans, tous les despotes coalisés contre notre sainte Liberté [...] qu'autant qu'il sera en notre pouvoir, nulle Bastille ne restera sur la terre, nul peuple dans les fers ; que tous les hommes trouveront en nous des frères, et tous nos concitoyens des soutiens inébranlables de la République française, une et indivisible. Nous le jurons sur les ruines de la Bastille ; nous le jurons sur les droits immortels de l'homme et du citoyen... $\gg^{38}$.

Fait remarquable, ce discours accompagne alors une médaille de fer frappée à partir des chaînes de la Bastille offerte aux députés et autres autorités politiques du moment. De façon particulièrement efficace, Palloy parvient donc à continuer d'utiliser l'événement de 1789 pour soutenir sa position publique plusieurs années plus tard, faisant donc clairement de la démolition de la Bastille le point de départ de tout le processus révolutionnaire et le fondement même de la Liberté. Il est remarquable que les interventions de Palloy plébiscitant son action et l'action du peuple parisien lors de la journée du 14 juillet sont toujours accompagnées de la présentation d'objets « souvenirs » de la Bastille, nous invitant à insister sur cette « matérialité » attachée au discours construit par le démolisseur.

\section{La matérialité du souvenir : les objets au cour du discours}

Boulets extraits de la forteresse, pierres issues des ruines de la prison, Palloy tient clairement à associer à ses initiatives festives un support matériel, relais de ses commémorations. Il utilise ce procédé à grande échelle en multipliant les objets souvenirs de la Bastille, devenus autant de reliques patriotiques. Parmi les plus célèbres et les plus originaux, les maquettes de la Bastille que nous avons déjà mentionnées, modèles réduits de la prison, que le démolisseur décide de diffuser dans tous les départements de France, ou encore les plaques de pierres présentant le plan de la Bastille, distribuées dans les 244 districts. On touche ici à un des aspects les plus spectaculaires du personnage et de son entreprise ${ }^{39}$.

(38) Pierre-François PALloY, Serment républicain, BNF, 4- LB41- 292 (A). Comme de nombreux opuscules de Palloy, ce serment a été également placardé dans les rues de Paris.

(39) Sur l'entreprise de distribution des maquettes et des plans par Palloy: Jean-Pierre BABELON, Les Maquettes et les pierres de la Bastille. Récolement des souvenirs lapidaires provenant de l'activité du patriote Palloy, La gazette des Archives, $\mathrm{n}^{\circ} 51,4^{\mathrm{ème}}$ trim. 1965 (janvier 1966), p. 217-230 ; DEM M., « P. F. Palloy, l'entrepreneur qui démolit la Bastille », Bâtir, n 167, juin 1968, p 62-63 ; Charles Bouché et Thierry BoDIn, Décorations et souvenirs historiques, lettres autographes, manuscrits et documents ...archives de Palloy démolisseur de la Bastille, Vente à Paris, Drouot Richelieu, 19 mai 1989, S. 1., s. n., Dumousset-Deburaux, 1989 ; Victor FouRNEL, Le patriote Palloy et l'exploitation de la Bastille, Paris, Honoré Champion, 1892 ; LAURENT P., La Bastille et le patriote 
Rappelons ici que même si le mérite d'avoir organisé à grande échelle la création et la diffusion des maquettes revient à Palloy, il n'en demeure pas moins que l'idée d'un modèle réduit de la Bastille est de l'un de ses ouvriers, le tailleur de pierre nommé Dax. Dans un des registres conservés à la Bibliothèque historique de la Ville de Paris se trouve en effet le compte rendu de la cérémonie donnée à l'Hôtel de Ville le 23 février 1790. On y voit Palloy tenir par le bras « le nommé Dax qui avait ébauché la petite Bastille » avant que celui-ci fasse à son tour un petit discours dans lequel il proclame que c'était «la Liberté qui lui avait donné l'idée de sculpter ce petit monument $»^{40}$. Le premier modèle « officiel » est présenté le 2 septembre 1790 à l'Assemblée, accompagné du texte de la capitulation de la forteresse, de l'échelle de Latude et de sachets de poudre. Le discours que Palloy prononce alors devant l'Assemblée illustre bien sa volonté de mettre en avant son engagement en faveur de la Révolution, le lien qu'il veut indissoluble à ce moment entre le Roi et la Révolution par l'intermédiaire de la Bastille prise et abattue.

« Moi-même j’y suis entré un des premiers, mes ouvriers y combattaient avec moi. N'écoutant que l'amour de la patrie, j'allai sans en avoir reçu l'ordre, abattre ces tours, moi-même je les frappai le premier, j'armai la main de mes ouvriers ; l'Assemblée de la Commune et le roi de lui-même ont approuvé mon zèle... $»^{41}$.

Palloy multiplie les objets souvenirs. Boutons, encriers creusés dans les déchets de la forteresse, jeux de cartes et de dominos assurent la publicité d'une véritable boutique de souvenirs qu'il entend relayer auprès des plus hautes sphères politiques. Ainsi Palloy commande une poupée destinée à la fille du roi, Marie Thérèse, dont on fête le treizième anniversaire, fabriquée à partir des rideaux de la chambre du gouverneur De Launay. On le voit également offrir au Dauphin pour les étrennes de 1791 un jeu de dominos taillé dans le marbre de la cheminée du même gouverneur ${ }^{42}$. Les pierres de la Bastille serviront également à la fabrication de bustes de Rousseau

Palloy, ou notes inédites sur un modèle de la Bastille conservé aux archives des Ardennes, Reims, Imprimerie et lithographie Matot-Braine, 1889 ; Henri LEMOINE, Le démolisseur de la Bastille. La place de la Bastille, son histoire de 1789 à nos jours, Paris, Perrin, 1930 ; id., Les comptes de la démolition de la Bastille, Bulletin de la Société de l'Histoire de Paris, 56 année, 1929, p. 77-83.

(40) B.H.V.P., CP 5253, registre 4, p. 963-971, Compte rendu de la cérémonie à l'Hôtel de Ville.

(41) Archives parlementaires, Tome XVIII, p. 511.

(42) Le couvercle de cette boîte est aujourd'hui conservé au musée Carnavalet. Musée Carnavalet, OM 520. 
et de Mirabeau, qui seront offerts à l'Assemblée nationale, le 6 octobre 1791. C'est donc une réelle postérité, une seconde vie, qui est offerte aux matériaux de la défunte Bastille.

Il est à noter cependant que ces objets ne constituent pas uniquement une « illustration pittoresque » mais au contraire fondent le discours même de l'événement. Cette fonction de l'objet apparaît clairement dans la lettre écrite aux directoires des chefs lieux de départements qui accompagne les envois d'objets « commémoratifs » associés aux célèbres maquettes :

« Il ne m'a point suffit d'avoir aidé à renverser les murs de la forteresse, il fallait en perpétuer l'horreur de son souvenir. D'une Bastille j'en ai fait 83 dont j'ai fait hommage à chacun des départements, afin que ses ruines s'étendent pour ainsi dire sur tout la France et rappellent à jamais aux citoyens vertueux l'atrocité de nos despotes. Des pierres mêmes des cachots affreux j'ai reconstruit l'image du tombeau [...] et des dalles sur lesquelles ont péri tant de victimes je les consacre à porter l'empreinte du roi, l'auguste soutien de notre constitution $\gg^{43}$.

À la suite de ce texte apparait la liste des objets qui accompagneront l'envoi dans les départements. Parmi ceux-ci nous pouvons mentionner un plateau fait des débris de la Bastille sur lequel sera posée la maquette, une dalle provenant des cachots, un plan de la forteresse avec ses accessoires, un description exacte de la Bastille, un tableau représentant le tombeau sous lequel reposent les victimes trouvés mortes lors de l'assaut, un boulet et une cuirasse trouvés dans les murs de la Bastille, trois volumes de procèsverbaux des Électeurs parisiens, un tableau représentant le roi, Bailly, et La Fayette et les ouvrages de Dussault et Latude. Ainsi les objets jouent un rôle central dans la production du discours de Palloy et, bien loin d'être de simples accessoires, participent pleinement à la construction d'une première histoire de la Bastille, et par là même de la Révolution.

Nous retrouvons le même phénomène à travers la production et la diffusion de médailles conçues par Palloy. En effet, parmi les nombreux objets que Palloy utilise pour soutenir son entreprise de publicité du chantier, les médailles figurent parmi les plus originaux et les plus remarquables. Nous nous référons ici en particulier aux travaux d'Alain Weil ${ }^{44}$, expert en numismatique, et de Gilles Michaud qui nous donnent des informations

(43) BHVP, CP 5252.

(44) Alain WEIL, «Sur l'origine historique des métaux employés à la confection des médailles de Palloy », BSFN, décembre 1974, n¹0, p 692-694; id., Histoire et numismatique du patriote Palloy, Paris, SPES, Lyon, Antika 1, 1976. 
précieuses sur ces objets. Si l'on s'en tient aux travaux des numismates, il apparaît probable que Palloy ait fait frapper 45 coins originaux. À raison de 1000 exemplaires par coin, le petit nombre d'exemplaires participant à la valeur symbolique de l'objet, sa production serait d'environ 25000 médailles, puisque les plaques frappées sont presque toujours assemblées par paires.

Là encore nous retrouvons l'enjeu du matériau utilisé par le démolisseur et la force symbolique qui y est associée. On trouve en effet de façon récurrente des mentions telles que : «ce métal provient des verroux de la Bastille ${ }^{45}$, ou encore « Ce métal vient des chaînes du pont levi de la Bastille ${ }^{46}$, Palloy insistant ainsi sur l'origine du métal qu'il emploie. De même, dans le serment républicain qu'il avait coutume de joindre à ses envois de médailles, on peut lire :

«Délivré avec une médaille de fer provenant des chaînes de la Bastille, et donné par Palloy, à ses amis républicains $\aleph^{47}$.

La question de la véritable origine de ces métaux a nourri la polémique. Cependant, comme le souligne Alain Weil, la correspondance de Palloy, où il est à plusieurs reprises question de la recherche de matériaux chargés d' « émotion » historique, semble indiquer que les médailles étaient effectivement fabriquées à partir de matériaux spécifiques. Parmi les thèmes représentés sur ces médailles, celles commémorant la prise et la démolition de la Bastille sont très largement représentées. À l'occasion de la conception de ces médailles, Palloy participe activement à la création d'un champ iconographique et lexical inédit. Une fois encore l'objet est au cœur de la création du sens et dépasse largement la fonction de simple illustration ou de « souvenir ».

À ce titre, les médailles de Palloy connaissent deux phases essentielles. La première s'étend de l'été 1789 à la fin de l'année 1792, alors que la deuxième couvre la période allant de 1793 au début du XIX ${ }^{\mathrm{e}}$ siècle. Alors que les rares médailles de la deuxième période ont seulement pour fonction d'assurer à Palloy sa survie politique, la première période est quant à elle bien plus riche dans la mesure où elle constitue le cœur du projet du démolisseur. Ces médailles de la première période, celles de la monarchie constitutionnelle et des tous premiers temps de la république, peuvent être partagées en trois groupes.

(45) Michel HENNIN, op.cit., référence 74.

(46) Ibidem, référence 360.

(47) BNF département des manuscrits, NAF 3242, f². 
Le premier concerne les médailles qui reprennent l'iconographie traditionnelle comme par exemple les emblèmes des trois ordres (épée, crosse et bêche surmontée d'un bonnet de la liberté croisés et entourés de lauriers). Le deuxième regroupe l'ensemble des médailles figurant la colonne dont Palloy a reçu la commande et qui doit être érigée sur la place de la Bastille désormais arasée. Le troisième ensemble enfin, le plus riche et le plus intéressant, rassemble toutes les médailles que Palloy fait frapper selon l'évolution de la situation politique. Celles-ci peuvent concerner aussi bien la fidélité « Au bon roi Louis XVI » que la louange aux galériens de Châteauvieux amnistiés « Donnée sur les décombres de la Bastille le 9 avril 1792 lan 4 de la liberté par Palloy patriote aux soldats de Châteauvieux victimes du despotisme reconnus innocents par un décrets du 30 décembres 1791 sollicité par Collot d'Herbois ».

Or la variété des circonstances n'oblitère pas l'unité de l'iconographie marquée par l'omniprésence de la Bastille en cours de démolition comme si, quelle que soit l'époque, la Bastille restait le fondement ou le référent indispensable de la Révolution en train de se faire, sorte de source originelle de tous les événements en cours et à venir. Omniprésence d'autant plus marquante que la Bastille est non seulement démolie en image mais concrètement abattue dans la main même de celui qui en manie un vestige, comme l'indique par exemple «Ce plomb scellait les anneaux qui enchainoient les victimes du despotisme », «Législateurs ce métal provient des chaînes de notre servitude que votre serment du 20 juin 1789 a fait briser le 14 juillet suivant, par Palloy patriote ».

De façon tout à fait originale, Palloy invente donc en quelque sorte un nouveau personnage, la Bastille, sorte d'allégorie de la «Liberté conquise » à la différence de la plupart des médailles frappées durant la période qui font référence à des personnages bien réels. En outre, héritier d'une culture classique Palloy utilise dès avant 1792 toute une série de symboles promis à un bel avenir : le coq qui surveille, le faisceau qui lie les citoyens, le bonnet symbole de la liberté, le lion symbole de la force. Si bien que l'iconographie des médailles permet à Palloy à la fois d'entrer dans le discours révolutionnaire mais aussi de le guider afin d'y inscrire de façon définitive la place de la forteresse.

Enfin, il convient ici de bien rappeler la gratuité des dons organisés par Palloy, en particulier pour les maquettes de la Bastille. Nous savons en effet que le Sieur Pommay a fabriqué et vendu de nombreux modèles en plâtre, cependant les maquettes sorties de l'atelier de Palloy bénéficient d'une reconnaissance particulière parce qu'elles sont, quant à elles, offertes en hommage et qu'elles constituent par leur matériau même, une incarnation 
de la forteresse honnie et désormais anéantie. Ces dons sont donc au cœur du discours du démolisseur et font partie intégrante de la construction de la mémoire, dépassant largement le statut de souvenir «illustratif », d'autant qu'ils semblent faire l'objet d'une entreprise de communication complexe mettant en œuvre des stratégies de « don-contre don » à grande échelle afin de répondre à une stratégie d'affirmation de Palloy sur la scène publique.

\section{Palloy et les dons, stratégie de prise de pouvoir ?}

Afin de mener à bien son entreprise de diffusion du souvenir de la Bastille, Palloy crée un groupe d'une grande originalité : les « Apôtres de la Liberté », appelés aussi « Apôtres de Palloy », dont les membres sont chargés de porter les maquettes - et la bonne parole « paroissienne »-à travers la France :

«La mission des Apôtres a eu pour objet depuis l'époque de la Révolution française, le 14 juillet 1789, lors de la prise de la Bastille, de seconder P. F. Palloy, leur instituteur, dans tous les actes de patriotisme qu'il a manifestés constamment pour la propagation de la foi constitutionnelle et le maintien de la liberté $»^{48}$.

Au nombre d'une soixantaine, les apôtres - amis, parents, collaborateurs ou ouvriers de Palloy - forment une société, avec un règlement et un insigne apostoliques (une médaille faite avec les fers de la Bastille). Tous ces hommes ont pour tâche d'assister l'entrepreneur lors des cérémonies et des célébrations qu'il organise. Néanmoins, dix d'entre eux sont plus spécialement chargés d'aller porter dans les chefs-lieux des 82 départements (Seine exceptée) les 246 caisses qui contiennent les souvenirs de la Bastille. C'est à partir du mois d'octobre 1790 que ces hommes sont envoyés effectuer leurs tournées dans l'ensemble des départements tout nouvellement créés. Ces apôtres doivent prêter serment de fidélité à leur « maître » dont nous trouvons la trace aux archives de Paris :

«Le 14 [février 1790], les commis des bureaux de M. Palloy, voulant lui témoigner un attachement invincible, déclarent qu'il prêteront devant lui, à l'issue d'un repas patriotique qui aura lieu le surlendemain du paiement de leurs appointements, le serment prescrit par le règlement apostolique de

(48) BNF, département des manuscrits, Nouvelles acquisitions françaises (désormais NAF), 3241, fol. 140, « Tableau nominatif des citoyens qui composent l'association des Apôtres de la Liberté » 
lui rester à jamais fidèlement liés de cœur et d'esprit, et de le défendre et soutenir envers et contre tous $»^{49}$.

La diffusion des maquettes dans les départements prend la forme d'une véritable entreprise car il s'agit de déplacer plusieurs caisses qui contiennent entre autres, comme nous l'avons déjà souligné, le modèle de la prison avec ses accessoires (grilles, portes, inscriptions, l'échelle de Latude accrochée à une fenêtre de la tour du Trésor, ou encore une horloge marquant $5 \mathrm{~h} 30$, heure de la capitulation), un plateau de bois servant de base au modèle et une dalle de pierre sur laquelle est gravé le portrait du roi $^{50}$. Les dix Apôtres distributeurs partent en tournée au mois d'octobre 1790. Ils reçoivent de Palloy une livre par lieue de route et, une fois arrivés à destination ${ }^{51}$, neuf livres par journée de séjour. Si l'organisation matérielle est bien pensée, son cadre spirituel est particulièrement étonnant dans la mesure où, afin d'assurer la diffusion de son projet et de sa notoriété, Palloy réinvente en quelque sorte l'association entre Jésus et ses apôtres. Sorte d'étonnant Christ moderne il entend porter la bonne parole - sécularisée cette fois - de la Révolution en réutilisant des référents et des rites anciens afin de diffuser un discours totalement nouveau. $\mathrm{Ce}$ procédé se retrouve également à l'occasion de cérémonies, notamment lors du transport des cendres de Voltaire, le 11 juillet 1791, à l'occasion duquel Palloy fait processionner une maquette de la Bastille comme on faisait traditionnellement circuler les reliques religieuses.

Dans cette entreprise menée conjointement par Palloy et par ses Apôtres, le caractère gratuit des multiples hommages rendus aux pouvoirs locaux est rappelé à maintes reprises, le démolisseur étant particulièrement soucieux de répéter à ses hommes qu'en aucun cas ils ne doivent accepter une quelconque rémunération, quelle qu'en soit la forme. Il s'agit bien sûr d'éviter toute accusation éventuelle de corruption et de rémunération douteuse de Palloy et de ses hommes. C'est un acte gratuit que Palloy entend faire en distribuant les souvenirs de la maquette. Il souhaite ainsi se prémunir contre toute attaque et contre toute ombre qui pourrait être portée sur son engagement patriotique. En outre, ces dons doivent faire

(49) Archives de Paris, 4 AZ 719. Collection Boret, Papiers de Palloy.

(50) Pour la liste des objets présents dans les caisses, voir Archives de Paris, 4 AZ 719 et BNF, département des manuscrits, NAF 3241, f $^{\circ} 119-129$.

(51) Archives de Paris, 4 AZ 719. Pour avoir une description de ce que pouvait être un tel voyage, voir Gustave BORD, « Journal d'un Apôtre de la Liberté », Revue de la Révolution, 1886, Tome I, p. 168-177. Cet apôtre aurait dépensé, en dix mois, 1566 livres de séjour, 2556 livres de voyage, et aurait reçu de Palloy 1340 livres. 
l'objet d'un rituel rigoureusement orchestré pour lequel le démolisseur donne des consignes claires. Ainsi l'apôtre Legros rend compte également de la cérémonie qui a eu lieu à Melun, le 5 décembre 1790 :

« Dans la grande salle de l'évêché l'on fit élever une espèce de piédestal pour poser l'effigie du modèle de la Bastille ainsi que les autres objets qui composaient l'envoi. Après quoi le département en corps et la municipalité et le district sont entrés accompagnés de la garde nationale. Chacun ayant pris place, Monsieur le président du Département fit part à l'Assemblée de la lettre de M Palloy et de mon pouvoir, après lequel le président me remit la parole afin de prononcer mon discours. Ce discours prononcé l'on dressa le procès-verbal que j'ai signé, dont $M$ Palloy est nanti. Le peuple a beaucoup applaudi au patriotisme de M Palloy $»^{52}$.

Palloy s'engage donc clairement dans une stratégie de dons destinés à tous les personnages importants de la vie politique - et plus largement publique - de l'époque : monarque, députés et huissiers de l'Assemblée nationale, membres des institutions politiques locales...

Ainsi la stratégie d'affirmation d'un pouvoir menée par Palloy répondrait en partie aux logiques d'influence associées à ce type spécifique de relation qu'est le don, notion étudiée à la fois par les anthropologues et les sociologues ${ }^{53}$.

Comme le rappellent Gunter Gebauer, et Christophe Wulf ${ }^{54}$, dans les relations réciproques et ritualisées de dons se constituent des ordres sociaux et symboliques. Donner et recevoir régulent les situations de proximité et de distance, structurant le sentiment d'appartenance, mais aussi l'admiration, ou l'inimitié. Leur caractère rituel joue un rôle particulier dans la production de leur sens imaginaire et symbolique. Les cadeaux, d'un point de vue anthropologique et sociologique

« [...] sont l'expression d'une « dépense improductive » par laquelle le donneur renonce à un objet qui lui appartient et l'offre comme une part de lui-même à un destinataire. Le donneur montre sa disposition à renoncer à

(52) BHVP, CP 5252, Correspondance avec les apôtres, fol 78. Lettre signée de Legros, Lyon, décembre 1790 .

(53) La place centrale tenue par ces objets au sein des stratégies de prise de parole - et de pouvoir - par Palloy pourrait être analysée au prisme des analyses récentes effectuées en sociologie cognitive, domaine étudiant les comportements au travers des interactions entre les individus. Voir par exemple Bernard CONEIN, «L'action avec les objets. Un autre visage de l'action située ? », Raisons pratiques, $\mathrm{n}^{\circ} 8,1997$.

(54) Gunter GEBAUER, Christophe WULF, Jeux, rituels, Gestes, Les fondements mimétiques de l'action sociale, Paris, Anthropos, 2004. 
l'utilisation personnelle de son don et à assumer la perte de ce bien dans l'intérêt de celui à qui il l'offre. Par le cadeau offert, le donneur se montre généreux, large et respectable. Le destinataire lui offre la possibilité de se produire dans ce rôle de donneur et de se montrer dans cette mise en scène $»^{55}$.

Allons plus loin, le don peut même apparaître comme une stratégie visant à « inférioriser un donataire qui ne peut rendre la réciproque $»^{56}$. Ainsi les dons répétés organisés par Palloy à travers la France et à Paris même, caractéristiques des dons patriotiques de la période révolutionnaire, peuvent apparaitre, par la multiplication des rituels et des «types» de destinataires, comme une entreprise d'affirmation, sinon d'un pouvoir, au moins d'une influence nouvelle. En l'occurrence les objets jouent un rôle essentiel dans la stratégie d'affirmation de Palloy et de ses discours. Cette stratégie est couronnée de succès de 1789 à 1793, « âge d'or » d'un Palloy qui parvient à se faire connaître et reconnaître, à s'imposer par ses discours et ses fêtes sur la scène publique parisienne. Cette reconnaissance se traduit par l'attribution de chantiers à la charge politique et symbolique forte comme celui de l'aménagement de la prison du Temple pour accueillir la famille royale, ou celui des travaux après l'incendie du château des Tuileries - façon pour un gouvernement sans-culotte de mettre en valeur un sans-culotte dans une démarche de soutien des valeurs révolutionnaires. Notons néanmoins que cette stratégie trouve ses limites à partir de l'hiver 1793-1794 quand Palloy se trouve accusé de détournements de fonds et d'enrichissement abusif, accusations dont il sortira d'ailleurs lavé quelques semaines plus tard.

Ainsi, Palloy parvient par divers biais à affirmer pendant quelques années son influence. Il ne semble pas anodin qu'un tel personnage parvienne à s'imposer sur la scène publique comme il le fait et réussisse, en quelques semaines, à s'insérer parmi les groupes et les réseaux déterminants de la vie parisienne. À ce titre, Palloy semble mettre à jour de nouveaux modes de communication et, par là même, une nouvelle façon de faire de la politique, ce que Lynn Hunt a étudié comme étant une des grandes nouveautés de la période révolutionnaire.

(55) Ibidem, p. 101.

(56) Lucien FAGGiON, Laure VeRdON (dir.), Le don et le contre-don, coll. « Le temps de l'histoire », Université de Provence, 2010. 


\section{Une nouvelle stratégie de communication?}

C'est à travers les objets, les rites et les discours que Palloy parvient à affirmer sa position et à assurer son ascension sociale. Ce sont précisément ces aspects de la communication que Lynn Hunt analyse dans son ouvrage Politics, Culture and Class in the French Revolution ${ }^{57}$. L'auteure insiste sur la place centrale du langage, des symboles, des habitudes et des comportements pour comprendre ces processus de construction d'une « culture politique » :

« Through their languages, images, and daily political activities, revolutionaries worked to reconstitute society and social relations. [...] In the process, they created new social and political relations and new kinds of social and political divisions [...] The political culture of revolution was made up of symbolic practices, such as language, imagery, and gesture $»^{58}$.

Elle ajoute, en outre, qu'en conséquence d'un déplacement continuel de l'autorité politique pendant cette période, le charisme, qui ne peut donc plus seulement se situer dans un personnage, se trouve investi dans les mots. Le langage révolutionnaire est donc investi d'une autorité sacrée, ce qu'on retrouve, tel un leitmotiv, dans les discours de Palloy.

Or ce langage n'est pas confiné à l'expression verbale mais s'étend à de nombreux modes d'expression symboliques. Au cours de la Révolution, même les objets les plus ordinaires deviennent des symboles politiques et des sources possibles de polémiques. Ainsi les formes rituelles sont aussi importantes que leur contenu puisque ces symboles politiques et ces rites ne sont plus seulement des métaphores du pouvoir mais aussi les moyens et les fins du pouvoir lui-même.

La nouvelle communauté que veulent fonder les révolutionnaires doit passer par la régénération dans tous les aspects de la vie quotidienne, si bien que le politique se voit en quelque sorte investi partout, et tous les jours. De cette manière, la Révolution a largement augmenté le nombre de lieux où peut s'exercer le pouvoir, et a multiplié les tactiques et les stratégies pour le contrôler. Lynn Hunt fait ainsi référence à Michel Foucault et à son

(57) Lynn Hunt, Politics, Culture and Class in the French Revolution, University of California Press, 1984.

(58) Au travers de leurs pratiques du langage, du recours aux images et de leurs activités politiques quotidiennes, les révolutionnaires ont redéfini la société et les relations sociales. Ils sont ainsi parvenus à créer des liens et des groupes sociaux et politiques d'un nouveau genre. La culture politique de la Révolution a été pétrie de ces pratiques symboliques, dans le domaine du langage, de la production iconographique et des gestes. Ibidem., p.11-13 
idée d'un exercice du pouvoir qui s'impose hors de la sphère politique. À ce titre, la Révolution apparaît comme le moment privilégié d'un processus dans lequel la mobilisation politique se joue dans les clubs, les sociétés populaires et les journaux plutôt que dans les chaînes régulières et officielles du gouvernement.

Toutes les actions et postures (prendre la parole dans un club, porter la cocarde ou une bannière, prononcer un discours...) convergent pour produire une citoyenneté républicaine et un gouvernement légitime. Le pouvoir, par conséquent, apparaît comme un ensemble complexe d'activités et de relations. Les réseaux (familiaux, professionnels, de voisinage) deviennent ainsi indispensables pour pouvoir exercer une influence sociale et politique. Comment ne pas voir dans la constitution du groupe des Apôtres de Palloy une parfaite illustration de ce phénomène ? Enfin, Lynn Hunt développe la notion d' « outsider », pour insister sur la marginalité (par rapport à la structure sociale globale) comme aptitude particulière à endosser le rôle de « passeur social » et de « passeur de pouvoir». Elle remarque que vivre en certains lieux ou occuper des positions socioculturelles particulières augmentent la capacité à l'action politique. En effet, la plupart des militants politiques vivent aux marges, ou occupent les espaces périphériques de l'ancienne élite, au croisement de plusieurs groupes. Là encore, la figure de Palloy semble paradigmatique de ce phénomène, ce dernier se situant au cœur de ces nouveaux mécanismes, parfaitement représentatif de ces modes de fonctionnement et de l'invention de ces nouvelles formes de discours, et partant, de pouvoir.

En l'occurrence la notion de réseau semble essentielle pour comprendre la stratégie mise en œuvre par Palloy. Ainsi, le groupe des apôtres, par la diversité de son recrutement (Vainqueurs de la Bastille, membres de la garde nationale, monde politique, collaborateurs sur le chantier, hommes de lois, architectes, « bourgeois de Paris », artistes) permet à Palloy d'affirmer sa position sociale. Les pouvoirs se mêlent, économiques, techniques et politiques. L'entrepreneur bascule du côté du monde politique, sans pour autant abandonner celui du bâtiment. Sans négliger la place tenue par les «attributs » constitutifs du capital social, culturel et économique du démolisseur, la notion de réseaux est indispensable ici pour comprendre les mécanismes à l'origine de l'affirmation d'un homme. En temps de crise politique et sociale, on ne peut appréhender le pouvoir uniquement dans les charges officielles ou dans le champ du politique pur. Entre un pouvoir strictement politique en pleine mutation et un pouvoir économique plus traditionnel, Palloy semble vouloir - et pouvoir - profiter de la période révolutionnaire pour construire une forme nouvelle d'influence politique 
en s'appuyant sur des réseaux traditionnels qu'il se donne pour tâche de renouveler. À travers la création de sa figure publique - dès les premières semaines il se donne une sorte de nom de scène, « le Patriote Palloy »- le démolisseur semble donc mettre en œuvre de nouveaux procédés afin d'assurer son ascension sociale. Cette capacité nouvelle à « entrer en politique » a également été analysée par Haim Burstin, à travers le concept de « pragmatisme politique». Celui-ci tente de dévoiler les motivations qui peuvent pousser un individu pourvu d'un bon métier, d'une famille, à se lancer dans la politique. Il rappelle alors que, pour des hommes issus des professions libérales ou juridiques, l'héritage d'une éducation humaniste peut expliquer un tel engagement. Palloy, ayant suivi sa scolarité au collège d'Harcourt jusqu'en classe de $5^{\text {ème }}$, entrerait dans cette catégorie.

De manière plus générale, Burstin affirme que

« $[\mathrm{L}]$ a révolution engendre l'expérience collective d'un rapport tout à fait original avec le processus historique : la conscience de vivre un temps historiquement beaucoup plus dense et significatif que d'habitude. Cela est dû à la sensation qu'un nouveau rapport vient de s'instaurer entre une action et ses effets. Les circonstances apparaissent susceptibles d'être influencées par l'action individuelle ou collective. D'où l'effort des contemporains pour marquer leur nouvelle adhésion à l'événement par des actions significatives lors des échéances révolutionnaires, et en même temps leur aspiration à voir ce rôle publiquement reconnu. Les individus manifestent leur volonté d'inscrire durablement leur action particulière dans le devenir historique $»^{59}$.

Ce phénomène s'expliquerait par l'élargissement inédit de la participation des citoyens à la vie publique et à la politique, dans une société où le système de valeurs a été profondément bouleversé. Burstin fait alors référence aux personnages balzaciens humiliés par la société d'Ancien Régime, qui guettent le moment idoine pour s'affirmer. Nous pouvons penser que Palloy, dans une certaine mesure, entre dans cette catégorie d'individus qui cherchent à se mettre en valeur, non pas pour faire oublier un passé préjudiciable (nous n'avons aucune information de ce genre), mais pour mettre en place une nouvelle dynamique d'ascension sociale. Tout en évitant une lecture déterministe des logiques socio-économiques à l'œuvre, la Révolution a pu néanmoins apparaître à Palloy - sans lui nier

(59) Haim Burstin, L'invention des sans-culottes. Regards sur le Paris révolutionnaire, Paris, Odile Jacob, 2005, p. 114-115. 
pour autant toute conviction sincère pour les idées qu'il défend - comme l'occasion de se faire une notoriété et d'accéder à une reconnaissance qui ne lui aurait pas été permise dans le fonctionnement social et politique d'Ancien Régime. Il est remarquable à ce titre que Palloy (et le souvenir de la Bastille) se trouve évincé sous la Convention thermidorienne, régime cherchant à échapper au pouvoir sans-culotte dont Palloy serait la figure de proue symbolique, pour disparaître complètement lorsqu'il est décidé que la Révolution est achevée avec le Consulat puis l'Empire. Il faudra attendre la III ${ }^{\mathrm{e}}$ République pour que le souvenir de la Bastille réapparaisse, celle-ci souhaitant renouer avec l'idée d'un pouvoir «populaire».

C'est donc une nouvelle façon de faire de la politique qui apparaît alors, et à laquelle Palloy participe activement. À travers son exemple il semble que la vie politique se joue dans des lieux différents et selon des modes réinventés. La figure du démolisseur semble ainsi paradigmatique de ces nouveaux groupes qui peuvent accéder à la vie publique et au pouvoir. Dans cette entreprise, Palloy s'est appuyé sur le chantier de la Bastille et en a fait, de façon remarquablement efficace, un lieu incontournable. Par là même, Palloy est devenu un personnage de premier plan, sans pour autant figurer dans aucun parti, club ou autre appartenance « traditionnelle».

Ainsi Palloy est le prototype de ces nouveaux acteurs, qui par l'intermédiaire de procédés et de supports réinventés prennent la parole en saisissant l'occasion offerte par la Révolution. En l'occurrence, la genèse de la symbolique de la Bastille trouverait en partie ses racines dans cette prise de parole d'un genre nouveau, par un personnage qui parvient à saisir un moment particulier pour le hisser au rang d' «Événement », véritable pierre de touche d'un premier discours sur la Révolution. Ce discours et cette pratique mémoriels esquissés par Palloy peuvent expliquer en partie aujourd'hui le caractère populaire et spontané de la fête nationale, qui reste, malgré les transformations de significations opérées par les régimes successifs, associée à l'idée de démocratie et de prise en main par le peuple de son destin. Ainsi cette prise de parole par des personnages « secondaires » comme Palloy a su perdurer malgré les diverses réécritures 
que les régimes successifs ont pu opérer quant à la forme à donner au souvenir du 14 juillet.

Héloïse BOCHER

Institut d'Histoire de la Révolution Française, Paris I heloisebocher@gmail.com

Lycée Camille Claudel, Place d'Anyama 77340 Pontault-Combault 